ARTIGO DE REVISÃO REVIEW ARTICLE

\title{
Vantagens custo-efetivas da utilização de ureteroscópios flexíveis descartáveis em comparação com o uso de ureteroscópios flexíveis reprocessáveis
}

\author{
Cost-effective advantages of using disposable flexible \\ ureteroscopes versus using reprocessable flexible ureteroscopes \\ Nathalia Pey Tournillon Sper ${ }^{1}$ \\ DOI: 10.21115/JBES.v13.n1.p55-65
}

\section{Palavras-chave:}

ureteroscopia, resistência à flexão, marketing, análise de custo-benefício, esterilização

\section{Keywords}

ureteroscopy, flexural strength, marketing, cost-benefit analysis, sterilization

\section{RESUMO}

Objetivo: Analisar o custo-efetividade da utilização de ureteroscópios flexíveis descartáveis em comparação com o uso de ureteroscópios flexíveis reprocessáveis. Métodos: Revisão integrativa de literatura realizada nos bancos de dados SciELO, LILACS e MEDLINE, utilizando os descritores "ureteroscopia", "resistência à flexão", "marketing", "análise de custo-benefício" e "esterilização", cujo objeto trata das vantagens custo-efetivas da utilização do ureteroscópio flexível descartável. Resultados: Trinta e cinco artigos foram encontrados, sendo quatro utilizados no estudo. Além disso, características de sete modelos de ureteroscópios flexíveis comercializados no Brasil mais conhecidos atualmente foram apresentadas. Os resultados foram baseados na análise conjunta dos artigos selecionados e características dos modelos de flexíveis apresentados e discutidos em duas categorias: a evolução dos ureteroscópios flexíveis e marketing mix - ureteroscópios flexíveis de uso único. Conclusão: Apesar da escassez de pesquisas que se aprofundem em custos de utilização de um ureteroscópio flexível de uso único, comparado a um reprocessável, são notórios os benefícios advindos das inovações agregadas a esses dispositivos, dando retorno positivo tanto para o profissional que faz seu uso deles quanto para o paciente, fazendo-se necessário analisar mais profundamente a possibilidade de migração da "cultura" de uso de ureteroscópios flexíveis reprocessáveis para os descartáveis.

\begin{abstract}
Objective: To analyze the cost-effectiveness of using disposable flexible ureteroscopes versus using reprocessable flexible ureteroscopes. Methods: Integrative literature review conducted in the SCIELO, LILACS and MEDLINE databases, using the descriptors ureteroscopy, flexion resistance, marketing, cost-benefit analysis, and sterilization, whose object deals with the cost-effective advantages of using the flexible ureteroscope disposable. Results: Thirty-five articles were found and four were used in the study. In addition, characteristics of seven models of flexible ureteroscopes commercialized in Brazil, that are currently better known, were presented. The results were based on a joint analysis of the selected articles and characteristics of the flexible models presented, and discussed in two categories: the evolution of flexible ureteroscopes; and marketing mix - flexible single-use ureteroscopes. Conclusion: Despite the scarcity of research that deepens the costs of using a flexible single-use ureteroscope when compared to a reprocessable one, the benefits arising from the innovations added to these devices are notorious, giving a positive return both to the professional who uses it and to the patient, making it necessary to further analyze the possibility of migration from the "culture" of using flexible reprocessable ureteroscopes to disposable ones.
\end{abstract}

\footnotetext{
Recebido em: 09/02/2021. Aprovado para publicação em: 14/04/2021.

1. Mestre em Enfermagem pela Universidade do Estado do Rio de Janeiro; enfermeira do trabalho na modalidade de Residência pela Universidade do Estado do Rio de Janeiro; MBA em Gestão de Saúde e Administração Hospitalar; MBA em Marketing pela Universidade Estácio de Sá, Rio de Janeiro, RJ, Brasil.

Financiamento: Não houve financiamento para a elaboração deste estudo.

Conflito de interesses: A autora é funcionária da Boston Scientific do Brasil. Este material é decorrente de Trabalho de Conclusão de Curso de MBA em Marketing na Universidade Estácio de Sá, RJ. Não há conflito de interesses.

Autor correspondente: Nathalia Sper. Rua das Laranjeiras, 457, 704 A, Laranjeiras, Rio de Janeiro, RJ, Brasil. CEP: 22240-005. Telefone: +55 (21) 99011-7006. E-mail: nathy.sper@globo.com
} 


\section{Introdução}

O tratamento de patologias do trato urinário superior, especialmente as relacionadas a cálculos renais, muitas vezes se dá de forma cirúrgica, por meio de litotripsia com a utilização de ureteroscópios flexíveis, por ser menos invasiva do que, por exemplo, a realização de uma nefrolitotripsia percutânea - NLPC (Türk et al., 2016).

A utilização de ureteroscópios flexíveis analógicos reprocessáveis ainda é amplamente empregada, ainda que seu custo de aquisição seja alto e sua duração/longevidade seja questionável (Somani et al., 2013).

Ainda, há de se considerar que a quebra/inutilização de um ureteroscópio flexível analógico implica demora para sua reposição e/ou reparos onerosos e, consequentemente, a necessidade de possuir mais de um dispositivo, de modo a evitar interrupções cirúrgicas (Marchini et al., 2019).

Por sua vez, as vantagens do produto descartável são diversas. Entre elas, pode-se citar vantagens durante o procedimento cirúrgico, como a deflexão real de pelo menos $270^{\circ}$ em ambas as direções, permitindo ótimo torque, bem como qualidade de imagem de alta resolução pelo fato, em geral, de serem digitais - superiores à tecnologia de fibra óptica -, e a extinção de riscos de infecção associados ao seu reprocessamento, bem como eliminação do custo de esterilização (Marchini et al., 2019; Pontes \& Brito, 2013).

\section{Litíase renal (cálculos renais)}

Cálculos renais são conhecidos popularmente como pedras nos rins e se formam pela materialização de cristais minerais no interior do sistema urinário. Vários são os minerais que formam tais cálculos, como, por exemplo, estruvitas (derivados de infecções), contudo, em sua maioria, são formados por oxalato de cálcio. Há, além disso, outros minerais como ácido úriCo, cistina, sulfato de indinavir, entre outros (Cunha et al., 2020).

Suas causas não são bem estabelecidas, no entanto há a implicação de um fator familiar na formação de cálculos. Além disso, pode-se relacionar sua formação também a infecções, transtornos bioquímicos ou do fluxo urinário, perturbações metabólicas sistêmicas, hábitos alimentares, baixa ingesta hídrica, alto consumo de bebidas alcoólicas e a algumas medicações (Cunha et al., 2020).

Dependendo de sua localização, a obstrução do trato urinário por cálculos renais pode causar como sintoma mais comum a cólica renal, cuja dor em geral é repentina, inesperada e intensa, localizada em um dos flancos, e pode se irradiar para a parte anterior baixa do abdômen (Sampaio \& Di Biase Filho, 2000).

Para seu diagnóstico, são realizados métodos de observação, como exames de urina e exames de imagens, tais como radiografia, ultrassonografia, urografia excretora, tomografia computadorizada e ressonância nuclear magnética (Sampaio \& Di Biase Filho, 2000).

\section{Evolução dos tratamentos}

Vários são os métodos de tratamento da litíase (cálculo) renal, desde os clínicos, como a terapia expulsiva, que faz uso de medicações que relaxam a musculatura ureteral de modo a facilitar a eliminação dos cálculos, até os cirúrgicos. A escolha do tratamento se dá de acordo com fatores relacionados ao paciente e ao tipo, tamanho e localização do cálculo (Camilo et al., 2015).

Quanto aos métodos mais comuns de tratamento cirúrgico, os mais conceituados recentemente, e por ordem de menor a maior invasão para o paciente, são: a litotripsia extracorpórea (LECO), a ureterolitotripsia endoscópica e a NLPC (Camilo et al., 2015).

Para efeitos de maior compreensão, a palavra litotripsia tem origem no grego, sendo "lithos" = pedra + "trîpsis" = esmagamento ou trituração. Ou seja, é o procedimento cirúrgico no qual se utilizam meios extracorpóreos, como ondas de choque (vibração), e intracorpóreos, como litotripsia ultrassônica, pneumática-balística e a laser, para fragmentar cálculos para eventualmente serem expelidos pelas vias adequadas (Litotripsia, 2020; Sampaio \& Di Biase Filho, 2000).

Como mencionado anteriormente, a LECO é um método menos invasivo, uma vez que é realizado por meio de ondas de choque de baixa frequência e alta energia, através da pele do paciente, com o objetivo de fragmentar os cálculos, facilitando sua eliminação espontânea. Sua vantagem se dá porque é um procedimento que pode ser realizado em ambulatório e sem anestesia; em contrapartida, a maior objeção para sua utilização é o fato de ser indicado apenas para cálculos menores que $2 \mathrm{~cm}$ e, preferencialmente, localizados na pelve renal ou em cálices superiores ou médios, com distância entre o cálculo e a pele do paciente bem próxima (Camilo et al., 2015; Freitas et al., 2004).

Em relação aos pacientes, a LECO não é indicada para obesos, pacientes com marca-passo e aneurismas de aorta abdominal, além de ser totalmente contraindicada para coagulopatas não tratados e pacientes com hipertensão arterial não controlada, obstrução urinária distal ao cálculo e grávidas. Entre as complicações mais frequentes, podem-se citar sangramento urinário e obstrução ureteral por fragmentos de cálculos (Camilo et al., 2015; Freitas et al., 2004).

Outro tratamento não tão invasivo (mais invasivo do que a LECO, pois necessita de internação e anestesia, e menos invasivo do que a NLPC) é a ureterolitotripsia endoscópica, que retira os cálculos através de endoscópios introduzidos na uretra, ou seja, finos tubos que possuem iluminação na extremidade e que são introduzidos na árvore urinária a partir da uretra, podendo ser ureteroscópios semirrígidos (utilizados para cálculos de ureter) ou flexíveis. Estes últimos são capazes de alcançar os rins e seus cálices de mais difícil acesso. Além disso, a evolução dos ureteroscópios flexíveis, com imagens digitais, entre outros benefícios previamente citados, e 
o desenvolvimento das fontes de fragmentação (como o uso de laser), viabilizou uma ampliação nos índices de remoção de cálculos, bem como uma diminuição na morbidade ocasionada pelo método, tornando-se, assim, uma opção mundialmente difundida (Camilo et al., 2015; Freitas et al., 2004).

Sua indicação é para pacientes que tenham mau prognóstico com LECO e/ou contraindicações à NLPC e ainda para cálculos de pequeno volume, impactados e em anatomia de difícil acesso. Suas complicações não são tão preocupantes, por isso é um tratamento de ampla escolha, visto que, na maioria das vezes, necessita apenas de terapêutica conservadora, como em casos de lesão de vasos pélvicos ou, ainda mais comum, na transposição do ureter (Sampaio \& Di Biase Filho, 2000).

Por fim, dos tratamentos cirúrgicos previamente citados, a NLPC é o mais invasivo, visto que se trata de um procedimento aberto no qual um tubo rígido, chamado nefroscópio, é inserido no rim através de uma incisão de cerca de $2,5 \mathrm{~cm}$ na pele do paciente, e através dele são retirados os cálculos. Sua indicação é para cálculos renais maiores que $2 \mathrm{~cm}$, múltiplos e de alta densidade. Em relação aos pacientes, indica-se principalmente para casos em que a LECO é contraindicada (Camilo et al., 2015). Em relação às complicações, deve-se atentar para a lesão vascular seguida de sangramento importante, devido à sua gravidade, podendo ser necessária transfusão sanguínea. Além disso, podem advir pneumotórax, derrame pleural, lesão pulmonar, urossepse e desequilíbrio hidroeletrolítico secundário à absorção de líquidos (Camilo et al., 2015; Sampaio \& Di Biase Filho, 2000).

\section{Ureterolitotripsia flexível}

Segundo Ribeiro (2015), a ureterolitotripsia flexível pode ser considerada o método cirúrgico mais realizado por urologistas em todo o mundo, devido à sua evolução, seja com a invenção de dispositivos que possibilitam maior facilidade de acesso ao rim (como a bainha para acesso ureteral) e captura de cálculos (baskets), ou com o aparecimento de novas fontes de energia que possibilitam a litotripsia dos cálculos. Além disso, o autor menciona o advento de ureteroscópios flexíveis mais evoluídos, mais finos, com maior deflexão/maleabilidade e melhor qualidade de imagem.

Como desvantagens, Ferreira et al. (2019) referem a grande probabilidade da necessidade do uso de cateter duplo J e de mais de uma sessão para a retirada total do cálculo, apesar de esta última afirmação poder ser controversa, uma vez que os autores afirmam também que a taxa de sucesso com apenas um único procedimento é superior a 90\%.

Ainda, Ribeiro (2015, p. 18-20) cita que "estudos mostram que o treinamento da ureteroscopia é importante neste processo de aprendizagem, porém o custo dos modelos de treinamento disponíveis é muito alto". Assim, a "confecção de um modelo anatômico preciso, de baixo custo, que permita o treinamento dos jovens urologistas para a realização da
URF é de grande auxílio nas instituições com médicos em treinamento" (Ribeiro, 2015, p. 20).

Ademais, a necessidade de amplo conhecimento da anatomia do sistema urinário influencia o manejo do ureteroscópio flexível, bem como o acesso aos cálices, o procedimento e consequente sucesso do tratamento (Ribeiro, 2015).

Por sua vez, Ferreira et al. (2019, p. 225) mencionam o fato de a ureterolitotripsia flexível ter uma "curva de aprendizagem pequena [...] e uma relação custo/efetividade bastante favorável".

Ainda em relação à anatomia do rim, o ureteroscópio flexível alcança o infundíbulo inferior com mais destreza, porém é necessário observar a angulação do aparelho para acessar os cálices menores (Ribeiro, 2015). E, "devido à sua flexibilidade, permite que o ureter superior, a pelve renal e os cálices sejam atingidos por via retrógrada e que cálculos localizados nestas posições sejam fragmentados ou removidos pela uretra, sem a necessidade de orifício ou corte" (Ferreira et al., 2019, p. 225).

Por fim: "Os parâmetros anatômicos analisados nos moldes tridimensionais e comparados às pielografias retrógradas mostram que o número e a orientação espacial dos cálices menores, o ângulo infundíbulo-caliceal e o comprimento infundibular podem ser considerados fatores anatômicos restritivos ao acesso do ureteroscópio aos cálices do polo renal inferior" (Ribeiro, 2015, p. 51).

Em relação ao ureteroscópio flexível analógico e reprocessável, Ferreira et al. (2019) discorrem sobre seu custo (elevado) e sua pequena durabilidade (cerca de 40 cirurgias).

Todos esses fatores reforçam a hipótese desta pesquisa de possivelmente haver vantagens custo-efetivas na utilização de um ureteroscópio flexível descartável, uma vez que, para o procedimento cirúrgico, aparentemente há uma maior deflexão e qualidade de imagem (por ser, em geral, digital); para os pacientes, há eliminação de infecções associadas (devido ao seu uso único); e quanto ao preço, há redução de custos associados à quebra/reposição, manutenção, obtenção de um segundo como backup e esterilização (Marchini et al., 2019; Pontes \& Brito, 2013).

\section{Marketing mix}

O marketing mix diz respeito às combinações de elementos que compõem as atividades de marketing, com base nos estudos de Neil Borden, em 1949 (Ribeiro, 2010).

A partir de então, tal expressão passou a ser conhecida no Brasil também como composto de marketing e se tornou a teoria mais aceita para efetivar atividades de marketing, sendo considerado como base fundamental para o marketing tático/operacional (Ribeiro, 2010).

Assim, Kotler (1998, p. 97) definiu o marketing mix como "o conjunto de ferramentas que a empresa usa para marketing no mercado-alvo". 
Por sua vez, mercado-alvo foi definido por McCarthy (1978) como um grupo de consumidores mais homogêneo possível, para os quais a empresa almeja chamar atenção.

A teoria de Borden foi aprimorada por Jerome McCarthy, professor da Universidade de Michigan, em 1978, definindo os quatro compostos do marketing (4 Ps do marketing), sendo eles: produto, preço, praça e promoção (Amaral, 2020).

Cada um dos 4 Ps do marketing estão interligados e são definidos como (Ribeiro, 2010; Neilpatel, 2020):

- "Produto" trata do que a empresa vende, seja físico ou um serviço, por exemplo. Deve se destacar dos concorrentes para conquistar clientes e abrange: variedade de produtos, qualidade, design, características, nome de marca, embalagem, tamanhos, serviços, garantias e devoluções;

- "Preço" refere-se ao custo para o cliente, ou seja, valor da venda. Precisa ser justo e ter um equilíbrio de forma que não prejudique a margem de lucro da empresa nem espante seus clientes, prejudicando o faturamento. Ele engloba: preço de lista, descontos, concessões, prazos de pagamento e condições de financiamento;

- "Praça" é o local onde o produto é ofertado, e a maior dica é a de ir até os clientes, não esperar que se encaminhem até o produto. Ela compreende: canais, cobertura, variedades, locais, estoque e transporte;

- "Promoção" aborda todas as ações utilizadas com o objetivo de anunciar sua marca e o que ela oferece, visando fazer com que o consumidor, ao ter necessidade dela, a escolha naturalmente. Envolve: promoção de vendas, propaganda, força de vendas, relações públicas e marketing direto.

\section{Métodos}

A partir desta breve contextualização teórica, tem-se como questão norteadora deste estudo: o custo-efetividade da utilização do ureteroscópio flexível descartável é válido?

Tem-se como assertiva que as vantagens da utilização de ureteroscópios flexíveis descartáveis se dão por evitar a aquisição de mais de um analógico como garantia de ininterrupção cirúrgica, além de cortar gastos com esterilização, manutenção e reparo deles.

Definiram-se como objeto deste estudo as "vantagens custo-efetivas da utilização do ureteroscópio flexível descartável", tratado em publicações científicas, e como objetivo a análise do custo-efetividade da utilização de ureteroscópios flexíveis descartáveis em comparação com o uso dos ureteroscópios flexíveis reprocessáveis.

Este trabalho justifica-se por existir uma incipiência de estudos relacionados ao tema custo-efetividade desse tipo de produto, podendo ser um fator positivo para a expansão do marketing dos ureteroscópios flexíveis descartáveis.
Este estudo caracterizou-se como uma pesquisa descritiva, do tipo revisão integrativa. As pesquisas descritivas narram os fatos e fenômenos de certa realidade (Gerhardt \& Silveira, 2009). A revisão integrativa proporciona a síntese de conhecimentos e a incorporação na prática de conclusões de estudos reconhecidos cientificamente, devido ao rigor metodológico e aos achados relevantes, e incorpora seis fases para a sua elaboração (Mendes et al., 2008), as quais estão descritas a seguir.

Na primeira fase, identificaram-se o tema e a questão de pesquisa: o que há publicado nas bases de dados sobre ureteroscópios flexíveis descartáveis?

A seguir, foram estabelecidos os critérios para inclusão e exclusão de estudos na análise desta pesquisa, caracterizando-se como a segunda fase desta pesquisa integrativa. Nessa perspectiva, o critério de inclusão foi: publicações eletrônicas inscritas nas bases de dados SciELO (Scientific Eletronic Library Online), LILACS (Literatura Latino-Americana e do Caribe em Ciências da Saúde) e MEDLINE (Medical Literature Analysis and Retrieval System Online). Estabeleceram-se os seguintes critérios de exclusão: estudos duplicados e que não estivessem relacionados ao objeto desta pesquisa.

Ainda na primeira fase, elaborou-se um quadro comparativo para modelos de ureteroscópios flexíveis conhecidamente comercializados no Brasil, contendo as seguintes informações: empresa fabricante, modelo, deflexão, imagem, diâmetro externo, diâmetro do canal de trabalho, observações relevantes e esterilização ou uso único/descartável (Quadro 1).

A coleta foi realizada no mês de outubro de 2020, e os descritores selecionados foram: "ureteroscopia", "resistência à flexão", "marketing", "análise de custo-benefício" e "esterilização", retirados do sistema DeCS (Descritores em Ciências da Saúde), nos idiomas português, inglês e espanhol. O descritor "ureteroscopia" foi empregado na pesquisa pareado, utilizando o "AND", com cada um dos outros descritores mencionados.

$\mathrm{Na}$ terceira fase, os artigos foram avaliados a fim de se extraírem informações pertinentes das publicações selecionadas (categorização).

Na quarta fase, realizou-se a avaliação dos estudos incluídos na revisão integrativa, que foram agrupados em um quadro sinóptico (Apêndice A) com suas principais informações.

A seguir, os resultados foram interpretados - quinta fase - por meio da análise das colunas contidas no quadro sinóptico e das características dos modelos flexíveis apresentados, a fim de submeter à análise descritiva.

Por fim, a apresentação da revisão/síntese do conhecimento compôs a sexta fase, na qual as informações foram sintetizadas por meio de categorias, reunindo informações comuns às diversas publicações, utilizando-se, portanto, a lógica da aproximação de resultados. 


\section{Resultados}

A fim de obter informações mais precisas sobre os modelos de ureteroscópios flexíveis comercializados no Brasil mais conhecidos atualmente, foi realizada uma busca, e suas características foram indicadas no Quadro 1.
Ainda, outros ureteroscópios flexíveis de uso único foram encontrados na literatura, porém não constam no quadro acima por não serem comercializados no Brasil. São eles: Polyscope, SemiFlex, Pulsen, YouCare Tech YC-FR-A e Neoscope NeoFlex.

Quadro 1. Informações provenientes dos próprios fabricantes, relativas às características dos modelos de ureteroscópios flexíveis comercializados no Brasil mais conhecidos atualmente - 2020

\begin{tabular}{|c|c|c|c|c|c|c|c|}
\hline $\begin{array}{l}\text { Empresa } \\
\text { Fabricante }\end{array}$ & Modelo & Deflexão & Imagem & $\begin{array}{l}\text { Diâmetro } \\
\text { Externo }\end{array}$ & $\begin{array}{l}\text { Diâmetro } \\
\text { do Canal de } \\
\text { Trabalho }\end{array}$ & Observações Relevantes & $\begin{array}{l}\text { Esterilização ou Uso } \\
\text { Único/Descartável }\end{array}$ \\
\hline Karl Storz & FLEX-X ${ }^{2 S(1)}$ & $\begin{array}{l}270^{\circ} \mathrm{em} \\
\text { ambos os } \\
\text { lados }\end{array}$ & Fibras ópticas & $7,5 \mathrm{Fr}$ & $3,6 \mathrm{Fr}$ & Não informado & $\begin{array}{c}\text { Produto de múltiplos } \\
\text { usos (esterilização) }\end{array}$ \\
\hline \multirow[t]{2}{*}{ Olympus } & URF-V3 (2) & $\begin{array}{l}275^{\circ} \mathrm{em} \\
\text { ambos os } \\
\text { lados }\end{array}$ & $\begin{array}{l}\text { Digital de alta } \\
\text { resolução } \\
\text { compatível } \\
\text { com NBI } \\
\text { (Narrow Band } \\
\text { Imaging) }\end{array}$ & $8,4 \mathrm{Fr}$ & $3,6 \mathrm{Fr}$ & Não informado & $\begin{array}{c}\text { Produto de múltiplos } \\
\text { usos (esterilização) }\end{array}$ \\
\hline & URF-P7 (2) & $\begin{array}{l}275^{\circ} \mathrm{em} \\
\text { ambos os } \\
\text { lados }\end{array}$ & Fibras ópticas & $7,95 \mathrm{Fr}$ & $3,6 \mathrm{Fr}$ & $\begin{array}{l}\text { Design DuraBend que } \\
\text { oferece alta durabilidade }\end{array}$ & $\begin{array}{c}\text { Produto de múltiplos } \\
\text { usos (esterilização) }\end{array}$ \\
\hline \multirow[t]{2}{*}{ Richard Wolf } & $\underset{\text { (3) }}{\mathrm{BOA} \text { vision }}$ & $\begin{array}{l}270^{\circ} \mathrm{em} \\
\text { ambos os } \\
\text { lados }\end{array}$ & $\begin{array}{l}\text { Digital com } \\
\text { chip na } \\
\text { extremidade } \\
\text { distal }\end{array}$ & $\begin{array}{l}\text { 8,7 Fr/6,6 Fr } \\
\text { ponta distal }\end{array}$ & $3,6 \mathrm{Fr}$ & $\begin{array}{l}1 \text { canal de trabalho. } \\
\text { Tecnologia LED. Não precisa } \\
\text { de fonte e cabo de luz. } \\
\text { Compatível apenas com } \\
\text { processadoras de imagem } \\
\text { ENDOCAM Flex HD. }\end{array}$ & $\begin{array}{c}\text { Produto de múltiplos } \\
\text { usos (esterilização) }\end{array}$ \\
\hline & $\mathrm{COBRA}^{(3)}$ & $\begin{array}{l}270^{\circ} \mathrm{em} \\
\text { ambos os } \\
\text { lados }\end{array}$ & $\begin{array}{l}\text { Digital full HD } \\
\text { com chip na } \\
\text { extremidade } \\
\text { distal }\end{array}$ & $\begin{array}{l}8,7 \mathrm{Fr} / 5,2 \mathrm{Fr} \\
\text { ponta distal }\end{array}$ & $\begin{array}{c}\text { 3,6 Fr } \\
\text { canal de } \\
\text { trabalho e } \\
\text { irrigação/2,4 } \\
\text { Fr para fibras } \\
\text { laser até o } \\
\text { máximo de } \\
365 \mu \text { m e } \\
\text { irrigação }\end{array}$ & $\begin{array}{c}2 \text { canais de trabalho. } \\
\text { Tecnologia LED. Não } \\
\text { precisa de fonte e cabo } \\
\text { de luz. Compatível apenas } \\
\text { com processadoras de } \\
\text { imagem ENDOCAM Flex } \\
\text { HD. }\end{array}$ & $\begin{array}{c}\text { Produto de múltiplos } \\
\text { usos (esterilização) }\end{array}$ \\
\hline Russer & $\begin{array}{l}\text { Scorpion } \\
\text { R1 }\end{array}$ & $\begin{array}{l}275^{\circ} \mathrm{em} \\
\text { ambos os } \\
\text { lados }\end{array}$ & $\begin{array}{l}\text { Digital } \\
\text { com chip } \\
\text { CMOS na } \\
\text { extremidade } \\
\text { distal. } \\
\text { Resolução } \\
400 \times 400\end{array}$ & $\begin{array}{l}8,5 \mathrm{Fr} / 7,5 \mathrm{Fr} \\
\text { ponta distal }\end{array}$ & $3,6 \mathrm{Fr}$ & $\begin{array}{l}\text { Tecnologia LED. Não } \\
\text { precisa de fonte e cabo } \\
\text { de luz. }\end{array}$ & $\begin{array}{c}\text { Produto de múltiplos } \\
\text { usos (esterilização) }\end{array}$ \\
\hline $\begin{array}{l}\text { Boston } \\
\text { Scientific }\end{array}$ & $\underset{\text { (5) }}{\text { LithoVue }^{\mathrm{TM}}}$ & $\begin{array}{l}270^{\circ} \mathrm{em} \\
\text { ambos os } \\
\text { lados }\end{array}$ & $\begin{array}{l}\text { Digital com } \\
\text { chip CMOS na } \\
\text { extremidade } \\
\text { distal de alta } \\
\text { resolução }\end{array}$ & $\begin{array}{l}9,5 \mathrm{Fr} / 7,7 \mathrm{Fr} \\
\text { ponta distal }\end{array}$ & $3,6 \mathrm{Fr}$ & $\begin{array}{l}\text { Tecnologia LED. Não } \\
\text { precisa de fonte e cabo } \\
\text { de luz. Possui PC tudo em } \\
\text { um, touchscreen, que } \\
\text { inclui monitor, } \\
\text { processador } \\
\text { de imagens e controlador, } \\
\text { onde se acopla o } \\
\text { ureteroscópio flexível. }\end{array}$ & $\begin{array}{l}\text { Uso único/descartável } \\
\text { (sem necessidade } \\
\text { de reparos, } \\
\text { esterilização ou } \\
\text { comprometimento } \\
\text { cirúrgico) }\end{array}$ \\
\hline
\end{tabular}

Fonte: Elaboração própria, 2020.

(1) Disponível em: http://www.strattner.com.br/doc/artigos/ureteroscopio-flexivel-7\%2C5-fr-Flex-x2.pdf.

(2) Disponível em: https://www.olympus-europa.com/medical/en/Products-and-Solutions/Products/Urology/Ureteroscopes.html.

(3) Disponível em: https://www.richard-wolf.com/mam/data/Typo3/Prospekte/Urologie/D_696_BOA_COBRA_vision/D_696_BOA_COBRA_vision_V17_pt.pdf.

(4) Disponível em: https://www.russer.com/endoscopioflexiveldigital.

(5) Disponível em: https://allent.com.br/wp-content/uploads/2017/01/Uretroscopio-URO-350107-AB-LithoVue-Brochure-PrintResPT_ago16.pdf. 
Por fim, 35 estudos foram encontrados na revisão integrativa, de acordo com os descritores selecionados. Desses, quatro atenderam aos critérios de inclusão e exclusão desta pesquisa e, assim, suas principais informações foram organizadas em um quadro sinóptico, alocado no Apêndice A.

Aplicando-se o procedimento metodológico descrito anteriormente, foram selecionados quatro artigos para serem utilizados neste estudo, explicitados no Apêndice A.

O primeiro artigo do quadro sinóptico - "Análise de custos de reparos de ureteroscópio flexível: avaliação de 655 procedimentos práticos" - é uma pesquisa de campo quantitativa e descritiva que analisou os motivos mais comuns para reparos de ureteroscópios flexíveis (lentes turvas e fibras ópticas quebradas), bem como seus gastos por reparo e custos médios por procedimento, concluindo que essas despesas podem impactar significativamente a receita líquida de um procedimento (Kramolowsky et al., 2016).

A pesquisa intitulada "Entendendo os custos da ureteroscopia flexível" é uma revisão de literatura e discutiu os custos atrelados à ureteroscopia flexível. Afirma que um alto investimento inicial é necessário para a aquisição do ureteroscópio flexível e existem diversos custos atrelados (manutenção e reparo). Contudo, apesar desses custos, os autores asseveram que esse procedimento é lucrativo e eficaz, com custo-benefício superior em relação à litotripsia por onda de choque (Ziemba \& Matlaga, 2016).

Em "Análise de custo da ureterorrenoscopia flexível", os autores analisaram 100 procedimentos e concluíram que houve dano significativo na imagem do ureteroscópio flexível após a lavagem no $12^{\circ}$ procedimento. O reparo ocorreu após o $29^{\circ}$ e o $88^{\circ}$ caso; antes do segundo reparo, houve um declínio gradual no campo de visão. No momento da compra, o ureteroscópio custou $£ 15.000$ e cada reparo/troca custava $£$ 4.200. Portanto, o gasto total com o ureteroscópio foi de $£$ 23.400. Custos totais com equipamentos auxiliares foram de $£ 28.853$ ( $£ 23.053$ em descartáveis auxiliares e $£ 5.800$ em 10 sondas a laser), concluindo-se que procedimentos mais complexos requerem mais dispositivos auxiliares, aumentando o custo geral. Também consideraram que casos mais longos e complexos expõem o ureteroscópio a maior risco de danos. Por fim, afirmam que o advento de ureteroscópios mais duráveis pode, em última análise, reduzir a frequência de reparos caros e que todos os custos devem ser considerados (Collins et al., 2004).

Finalmente, a revisão sistemática de literatura "Uma compreensiva equação baseada na literatura para comparar a relação custo-eficácia de um programa de ureteroscopia flexível com dispositivos de uso único versus reutilizáveis" assegurou que ureteroscópios mais recentes oferecem eficácia clínica semelhante em relação aos reutilizáveis, com baixas taxas de complicações. Um dos estudos incluídos na revisão demonstrou contaminação em 100\% dos flexíveis após o processo de esterilização, porém nenhum estudo demonstrou menor taxa de infecção do trato urinário com o emprego de dispositivos descartáveis. Os autores recomendam o uso de tecnologia digital de uso único em casos de alto risco de danos ao ureteroscópio flexível, ou quando o preço para adquirir um novo ou para reparar for elevado, podendo, assim, dispositivos descartáveis ser mais econômicos. Além disso, alegam que os flexíveis de uso único podem ser bons para centros de aprendizagem. Por fim, mencionam como principais fatores envolvidos no custo de cirurgias com flexíveis: o preço de compra e a necessidade de reparo do ureteroscópio flexível; sua longevidade influenciada pela destreza do cirurgião, método de esterilização, tipo e local do cálculo, e instrumentação; despesas com reprocessamento ou reciclagem e despesas da sala de operação (Marchini et al., 2019).

Em relação às características dos ureteroscópios flexíveis comercializados no Brasil mais conhecidos atualmente, sete modelos foram expostos, no entanto os preços de compra deles não constaram no Quadro 1 desta pesquisa, pois é sabido que eles variam de acordo com o comprador (plano de saúde, hospital, paciente particular), bem como conforme as negociações (pacotes, aquisição de mais de um produto, entre outros).

A análise dos resultados do Quadro 1 indica que:

- Em relação à deflexão, foi pouca a diferença (apenas 50) entre os ureteroscópios apresentados, não sendo uma questão de importância relativa.

- Por sua vez, a qualidade da imagem de um dispositivo digital é extremamente maior, quando comparada com a de um dispositivo analógico, por possuir resolução infinitamente melhorada.

- Em relação ao diâmetro externo, percebe-se uma variação significativa entre os modelos, contudo esse parâmetro depende da escolha do cirurgião, uma vez que diâmetros maiores necessitam de bainhas maiores e maior dilatação ureteral, porém, ao mesmo tempo, permitem melhor irrigação. Por sua vez, diâmetros menores podem ser positivos para acessar anatomias complexas de forma atraumática. Contudo, apesar da significativa variação de diâmetro externo, deve-se atentar para a deflexão, muito semelhante em todos os dispositivos apresentados.

O diâmetro do canal de trabalho foi semelhante para todos os ureteroscópios flexíveis apresentados.

Entre as observações relevantes, as principais foram o modelo COBRA, da Richard Wolf, possuir dois canais de trabalho e a necessidade de aquisição de estação de trabalho própria, de modo a haver compatibilidade do uso do ureteroscópio flexível, como, por exemplo, a processadora de imagem ENDOCAM Flex HD, para os dispositivos da Richard Wolf, e o PC, para o dispositivo da Boston Scientific. No entanto, deve-se observar que, quanto aos ureteroscópios flexíveis da 
Richard Wolf, há ainda a necessidade de aquisição da câmera, enquanto o PC touchscreen da Boston Scientific, por ser tudo em um, dispensa torre de vídeo.

Por fim, o único ureteroscópio flexível apresentado como de uso único, ou seja, descartável, foi o modelo LithoVue ${ }^{\mathrm{TM}}$ da Boston Scientific.

\section{Discussão}

Para discussão dos resultados, elaboraram-se duas categorias baseadas na análise conjunta do Quadro 1 e do Apêndice A, que permitiram comparar as vantagens custo-efetivas da utilização de ureteroscópio flexível descartável em comparação ao uso do ureteroscópio flexível reprocessável.

\section{A evolução dos ureteroscópios flexíveis}

Com o desenvolvimento tecnológico, houve também desenvolvimento dos ureteroscópios flexíveis.

Duas características foram de grande notoriedade nos resultados deste estudo: a resolução digital de imagem e questões relacionadas ao reprocessamento dos ureteroscópios flexíveis.

A tecnologia digital vem sendo empregada como forma de melhorar a imagem e consequentemente permitir ao cirurgião maior e melhor campo de visão, tratando os cálculos com mais eficiência. Tal fato pode ser notado no Quadro 1, no qual dos sete ureteroscópios flexíveis apresentados, cinco possuem tecnologia digital.

Em relação à longevidade dos ureteroscópios flexíveis, alguns autores relatam que um digital é usado cerca de 21 vezes antes de ser necessário o seu reparo, enquanto os analógicos de fibra óptica são usados em média de 6 a 15 vezes (Karaolides et al., 2013). Ainda, além da maior longevidade dos digitais, alguns autores mencionam que o tempo cirúrgico é menor em 20\%, quando comparado com um analógico (Marchini et al., 2019).

No que concerne ao reprocessamento dos dispositivos, os danos aos ureteroscópios flexíveis ocorrem não apenas durante o procedimento, mas muitas vezes durante seu reprocessamento (esterilização), a depender do método utilizado e das técnicas empregadas (Marchini et al., 2019). Autores afirmam ainda que ureteroscópios flexíveis recondicionados duram menos do que novos, e os de uso único são mais resilientes que os reprocessáveis, inclusive em procedimentos de longa duração (Usawachintachit et al., 2017; Taguchi et al., 2018).

Assim, o custo total de um dispositivo reutilizável deve englobar compra, reparo e esterilização, sendo este último composto de mão de obra especializada, matéria-prima, maquinário, materiais para escovação, teste de vazamento e embalagem. Por sua vez, reparo e esterilização não devem ser somados na equação quando se tratar de um dispositivo de uso único, sendo seu principal fator o custo de aquisição (Marchini et al., 2019; Kramolowsky et al., 2016).
Martin et al. (2017) realizaram uma análise de custo dos ureteroscópios flexíveis reutilizáveis e concluíram que seu uso é favorável apenas após a realização de 99 procedimentos. Assim, sugeriram que, em termos de custos, o uso desse tipo de dispositivo é benéfico apenas em instituições com alto volume cirúrgico.

Por outro lado, Marchini et al. (2019), em seus estudos, verificaram, em sua revisão, que alguns estudos citam que, mesmo com os custos associados (danos, reparos, necessidade de dispositivo de backup, entre outros), os flexíveis reprocessáveis podem ser economicamente mais viáveis. Assim, o uso de flexíveis de uso único, relativamente aos reprocessáveis, somente é praticável dependendo de seu preço de compra (análise de número de casos multiplicado pelo preço de compra unitário do dispositivo).

Esses fatos fazem refletir sobre os possíveis efeitos benéficos da introdução de ureteroscópios flexíveis de uso único que vem ocorrendo no Brasil (Collins et al., 2004; Marchini et al., 2019).

\section{Marketing mix - Ureteroscópios flexíveis de uso único}

Um plano de marketing mix baseado no modelo dos 4 Ps, voltado para a área da saúde, com a finalidade de atraí-la ao mercado de novas tecnologias em dispositivos de saúde, em especial os ureteroscópios flexíveis de uso único, pode ser posto em prática de forma assertiva.

Em relação ao produto, este demonstrou ter atributos notoriamente positivos no que concerne tanto ao seu uso pelo cirurgião (ergonomia, imagem, deflexão, entre outros) como em relação ao paciente (mitigando taxas de infecção, reduzindo tempo cirúrgico e, por vezes, evitando a necessidade de mais de um procedimento).

Quanto à preço, há divergências. Alguns autores, como Marchini et al. (2019), alegam que o custo de obtenção do ureteroscópios flexíveis de uso único é alto, sendo somente praticável dependendo de seu preço de compra. Em contrapartida, outros estudos mencionam que a compra de ureteroscópios flexíveis reprocessáveis pode não compensar, a depender de sua longevidade e custos associados (Marchini et al., 2019; Kramolowsky et al., 2016). Ainda, sugere-se que os reprocessáveis só são válidos em instituição com grande volume cirúrgico (Martin et al., 2017).

Sobre a "praça", verificou-se que o modelo de ureteroscópio flexível de uso único apresentado neste artigo é de uma empresa estrangeira com sede no Brasil que faz vendas diretas e indiretas por meio de seus distribuidores. Assim, esse é um bom método para atender seus clientes, uma vez que há amplas formas para o consumidor final adquirir o produto o mais rápido possível.

Por fim, no que diz respeito à "promoção" do produto, percebe-se que, apesar de apenas um modelo constar na tabela, os ureteroscópios flexíveis de uso único vêm sendo introduzidos no Brasil e suas características e potencialidades 
têm sido apresentadas aos cirurgiões por meio de artigos em revistas brasileiras, como o de Marchini et al. (2019), eventos, como o curso teórico-prático (Stone Institute) citado por Coaracy (2018) realizado pela Sociedade Brasileira de Urologia (SBU) em parceria com a empresa fabricante, entre outros.

\section{Conclusões}

É notória a importância de analisar vantagens custo-efetivas na área da saúde, sendo evidente o crescimento de estudos relacionados à novas tecnologias incorporadas aos ureteroscópios flexíveis. Entretanto, há ainda escassez de pesquisas que aprofundem custos de utilização de um ureteroscópio flexível de uso único comparado a um reprocessável.

Ficam nítidos os benefícios advindos das inovações agregadas aos ureteroscópios flexíveis, dando retorno positivo tanto para o profissional que o utiliza quanto para o paciente. Nessa perspectiva, faz-se necessário analisar mais profundamente a possibilidade de migração da "cultura" de uso de ureteroscópios flexíveis reprocessáveis para os descartáveis. Por outro lado, há de se considerar que essa migração será mais rápida com a redução do preço de obtenção dos ureteroscópios flexíveis de uso único, podendo os custos ser semelhantes ao do uso de flexíveis reprocessáveis, quando considerados os custos associados a estes.

Nesta pesquisa, no entanto, pode haver questionamentos, na medida em que há a possibilidade da existência de modelos de ureteroscópios flexíveis que não constam no Quadro 1, mas que são utilizados no Brasil. No entanto, não foram achados registros de sua venda no país, por isso suas características não foram incluídas e comparadas.

Por fim, acredita-se que, em países com maior desenvolvimento técnico, científico e econômico, exista maior demanda de uso de ureteroscópios flexíveis descartáveis, considerando as notórias potencialidades em suas características, bem como por haver, a princípio, apenas fatores positivos quanto ao seu uso.

\section{Referências bibliográficas}

Amaral SA. Os 4Ps do composto de marketing na literatura de ciência da informação. Transinformação. 2000;12(2):51-60. Disponível em: https:// www.scielo.br/pdf/tinf/v12n2/04.pdf. Acesso em: 14 out. 2020.

Camilo G, Bouças RI, Achar RA, Achar E. Os principais tratamentos para a litíase renal. Rev Sci Health. 2015;5(3). Disponível em: http:// arquivos.cruzeirodosuleducacional.edu.br/principal/new/revista_ scienceinhealth/18_set_dez_2015/Science_03_2015.pdf\#page $=46$. Acesso em: 13 out. 2020.

Coaracy G. SBU-DF realiza eventos de educação continuada. Bol Urol. 2018;3(3):29-30. Disponível em: https://portaldaurologia.org.br/medicos/ pdf/bodau/Bodau_2018_03.pdf. Acesso em: 19 out. 2020.

Collins JW, Keeley FX, Timoney A. Cost analysis of flexible ureterorenoscopy. BJU Int. 2004;93(7):1023-6.

Cunha TS, Rodriguez A, Heilberg IP. Influência de disparidades socioeconômicas, temperatura e umidade na composição de cálculos renais. Braz J Nephrol. 2020;42(4):454-60. Disponível em: http://www.scielo.br/scielo.php?script=sci_arttext\&pid=S010128002020005022201\&lng=en\&nrm=iso. Acesso em: 13 out. 2020.

Ferreira PF, Madureira EM, Souza FL. Nefrolitotripsia flexível para tratamento de cálculo coraliforme completo: relato de caso. Rev Thêma et Scientia; 2019;9(1):222-30. Disponível em: http://www.themaetscientia.fag.edu.br/ index.php/RTES/article/download/1023/973. Acesso em: 13 out. 2020.

Freitas RM, Silva LC, Santos LJ, Tavares Júnior WC. Avaliação dos métodos de imagem no diagnóstico da urolitíase: revisão da literatura. Radiol Bras. 2004;37(4):291-4. Disponível em: http://www.scielo.br/scielo. php? script=sci_arttext\&pid=S0100-39842004000400014\&lng=en\&nrm= iso. Acesso em: 13 out. 2020.

Gerhardt TE, Silveira DT. Métodos de pesquisa. Porto Alegre: Editora da UFRGS; 2009.

Karaolides T, Bach C, Kachrilas S, Goyal A, Massod J, Buchholz N. Improving the durability of digital flexible ureteroscopes. Urology. 2013;81(4):71722. Disponivel em: https://www.goldjournal.net/article/S00904295(13)00044-7/fulltext. Acesso em: 19 out. 2020.

Kotler P. Administração de marketing: análise, planejamento, implementação e controle. 5 a ed. São Paulo: Atlas; 1998. 725p.

Kramolowsky E, McDowell Z, Moore B, Booth B, Wood N. Cost Analysis of Flexible Ureteroscope Repairs: Evaluation of 655 Procedures in a Community-Based Practice. J Endourol. 2016;30(3):254-6.

Litotripsia. In: Michaelis. Dicionário Brasileiro da Língua Portuguesa. São Paulo: Melhoramentos; 2020. Disponível em: https://michaelis.uol.com. br/moderno-portugues/busca/portugues-brasileiro/litotripsia/. Acesso em: 13 out. 2020.

Marchini GS, Torricelli FC, Batagello CA, Monga M, Vicentini FC, Danilovic $A$, et al. A comprehensive literature-based equation to compare costeffectiveness of a flexible ureteroscopy program with single-use versus reusable devices. Int Braz J Urol. 2019;45(4):658-70.

Martin CJ, McAdams SB, Abdul-Muhsin H, Lim VM, Nunez-Nateras R, Tyson $M D$, et al. The economic implications of a reusable flexible digital ureteroscope: a cost-benefit analysis. J Urol. 2017;197(3):730-5.

McCarthy EJ. Basic marketing: a managerial approach. 6a ed. Homewood, Illinois: Richard D. Irwin, Inc.; 1978. 752p.

Mendes KD, Silveira RC, Galvão CM. Revisão integrativa: método de pesquisa para a incorporação de evidências na saúde e na enfermagem. Texto Contexto Enferm. 2008;17(4):758-64.

Neilpatel [Internet]. O que são os 4 Ps do marketing? Tudo sobre o conceito + exemplos. 2020. Disponível em: https://neilpatel.com/br/blog/4-ps-domarketing/. Acesso em: 14 out. 2020.

Pontes RX, Brito FA. Perfil clínico de pacientes submetidos à litotripsia a laser por ureteroscopia flexível. 2013. Disponível em: http://imagens. devrybrasil.edu.br/wpcontent/uploads/sites/63/2014/12/23144038/ REVISTA-FACID-2013.1_VOLUME9__Numero01.pdf. Acesso em: 13 out. 2020.

Ribeiro BL. Estudo experimental da anatomia do polo inferior do rim aplicada à ureteroscopia flexível em moldes tridimensionais do rim humano e em pielografias retrógradas [Tese - Doutorado em Fisiopatologia e Ciências Cirúrgicas]. Rio de Janeiro: Faculdade de Ciências Médicas, Universidade do Estado do Rio de Janeiro; 2015. Disponível em: http://www.fisiocirurgiauerj.org/defesas/2015/Tese_ Bruno_Marroig.pdf. Acesso em: 13 out. 2020.

Ribeiro JF. Redes sociais nas empresas [Monografia - Bacharel em Comunicação Social com Habilitação em Publicidade e Propaganda]. Brasília/DF: Faculdade de Tecnologia e Ciências Sociais Aplicadas, Centro Universitário de Brasília; 2010. Disponível em: https://repositorio. uniceub.br/jspui/bitstream/123456789/1118/2/20700578.pdf. Acesso em: 14 out. 2020. 
Sampaio FJ, Di Biase Filho G. Litíase Renal. In: Bendhack DA, Damião R. Guia Prático de Urologia. 2000. cap. 18, p. 97-104. Disponível em: http:// transdoreso.org/pdf/Litiase_Renal.pdf. Acesso em: 13 out. 2020.

Somani BK, Al-Qahtani SM, Medina SD, Traxer O. Outcomes of flexible ureterorenoscopy and laser fragmentation for renal stones: Comparison between digital and conventional ureteroscope. Urology. 2013;82:1017-9.

Taguchi K, Usawachintachit M, Tzou DT, Sherer BA, Metzler I, Isaacson D, et al. Micro-Costing Analysis Demonstrates Comparable Costs for LithoVue Compared to Reusable Flexible Fiberoptic Ureteroscopes. J Endourol. 2018;32(4):267-73
Türk C, Petřík A, Sarica K, Seitz C, Skolarikos A, Straub M, et al. EAU Guidelines on Interventional Treatment for Urolithiasis. Eur Urol. 2016;69:475-82.

Usawachintachit M, Isaacson DS, Taguchi K, Tzou DT, Hsi RS, Sherer BA, et al. A prospective case-control study comparing LithoVue, a single-use, flexible disposable ureteroscope, with flexible, reusable fiber-optic ureteroscopes. J Endourol. 2017;31(5):468-75.

Ziemba JB, Matlaga BR. Understanding the costs of flexible ureteroscopy. Minerva Urol Nefrol. 2016;68(6):586-91. 


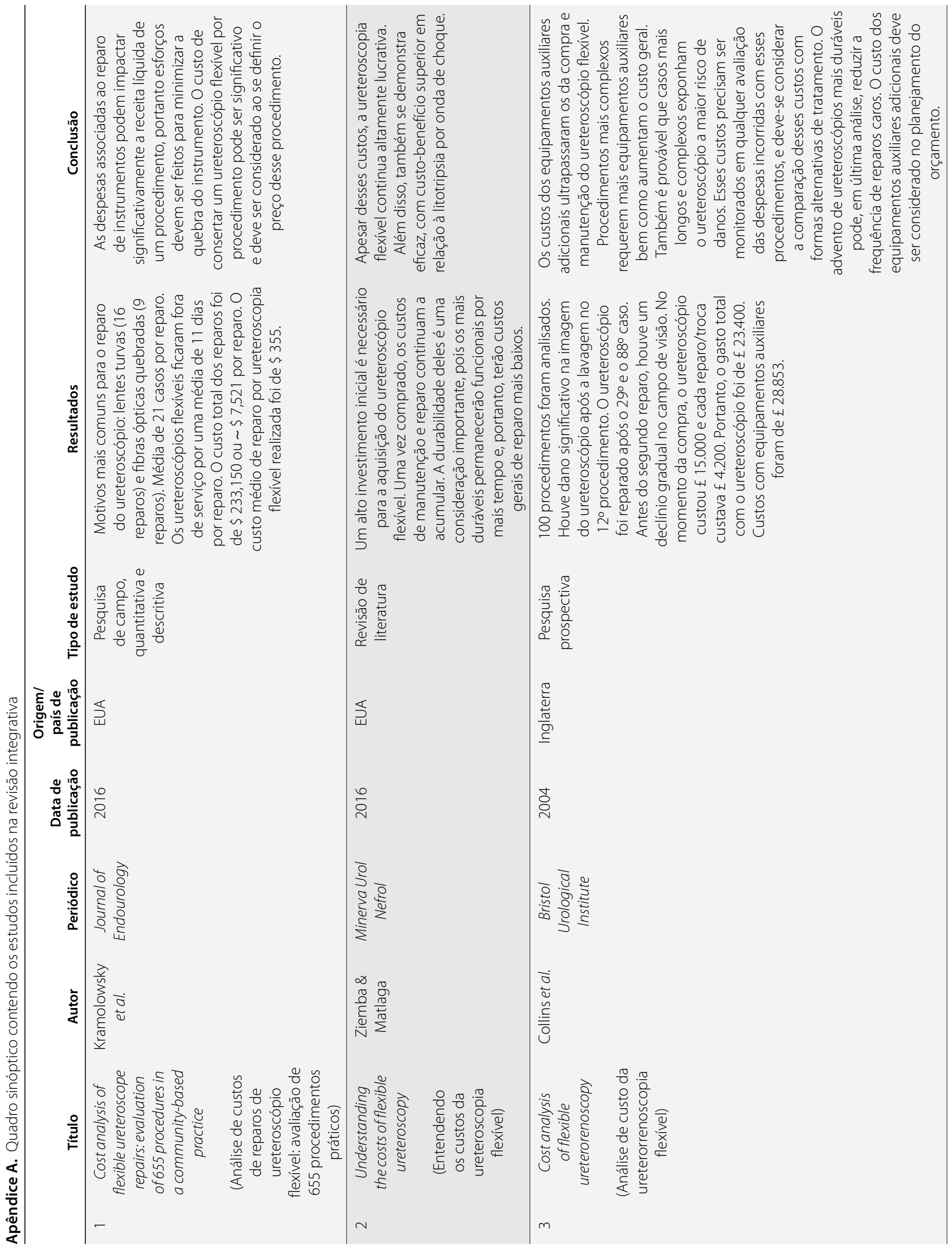




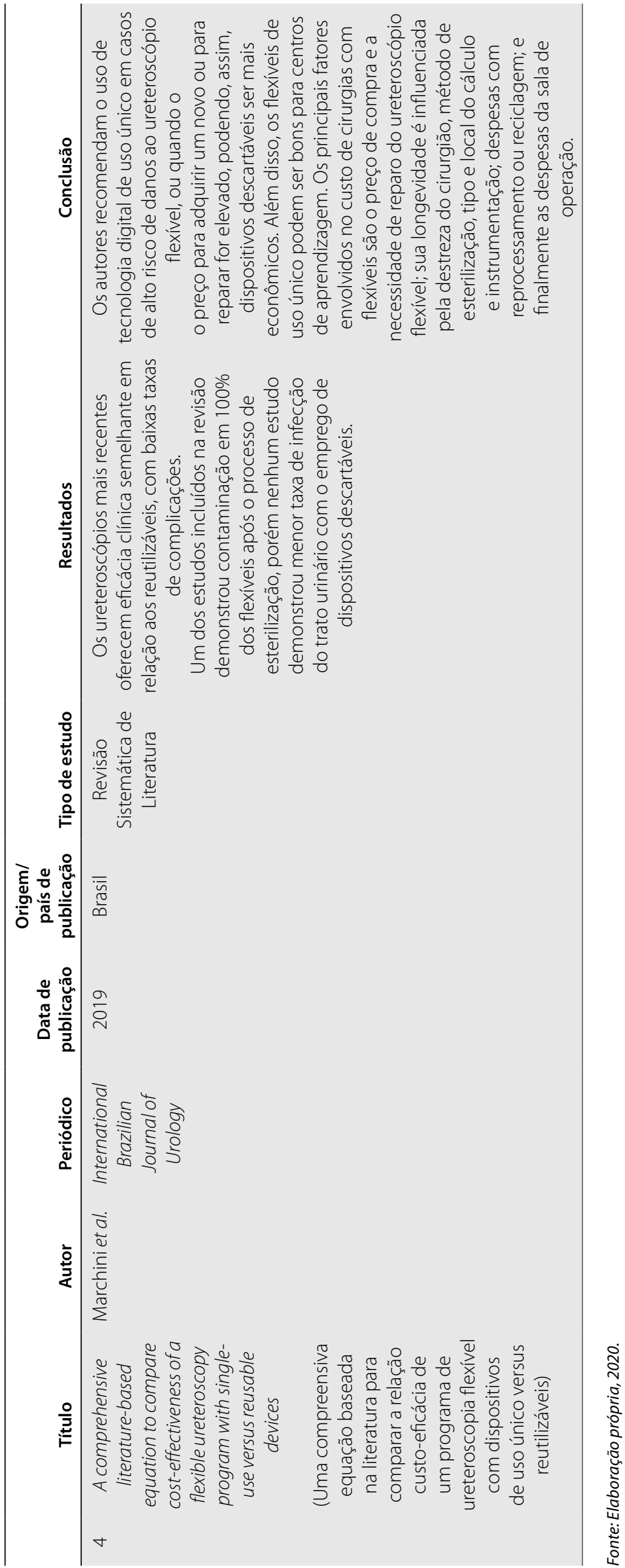

